\title{
Appropriate selection of patients with lumbar spinal stenosis for interspinous process decompression with the X STOP device
}

\author{
Carl Lauryssen, M.D. \\ Tower Orthopedics and Neurosurgical Spine, Beverly Hills, California
}

\begin{abstract}
$\checkmark$ With the aging of the population, the number of patients suffering from progressive lumbar spinal stenosis with symptomatic neurogenic intermittent claudication is projected to increase. Unfortunately, these patients are limited to a choice between nonsurgical conservative care and more invasive decompressive surgical procedures such as laminectomy with or without fusion. The X STOP interspinous process decompression system is a commercially available device that provides a minimally invasive alternative treatment, an intermediate option within the continuum of care for these patients. The X STOP is appropriate for patients with moderately severe functional impairment whose symptoms are exacerbated in extension and relieved in flexion. Implanted between the spinous processes without disrupting the normal anatomical structures, the X STOP limits narrowing of the spinal canal and neural foramina by reducing extension at the symptomatic level(s). In this report the author details the approved indications for use of the $\mathrm{X}$ STOP and discusses several illustrative cases.
\end{abstract}

KEY WORDS - spinal stenosis • interspinous process decompression • lumbar spine

\section{$\mathrm{L}$} UMBAR spinal stenosis is a narrowing of the spinal cord or neural foramina that occurs as a result of age-related spinal degeneration, particularly in the intervertebral disc and ligamentum flavum. ${ }^{2}$ The gradual loss of disc height in this syndrome causes the affected spinal segment to adopt a position of hyperextension, leading to bulging of the anulus, hypertrophy of the facets, spondylolisthesis, and thickening and calcification of the ligamentum flavum. ${ }^{2}$ Repetitive compression of the spinal canal and neural foramina produces root ischemia, which manifests clinically as neurogenic intermittent claudication. ${ }^{12}$ The hallmark clinical features include radicular symptoms that are provoked when the patient assumes positions that place the spine in extension, such as standing and walking. Conversely, symptoms are relieved when the patient assumes a position that flexes the spine, such as sitting or leaning forward; this is a differential diagnostic characteristic relative to other causes of neurogenic claudication. ${ }^{2,3}$

\section{Treatment Options}

There is a continuum of care for patients with neurogenic intermittent claudication associated with lumbar spinal stenosis that begins with conservative nonsurgical management. ${ }^{3}$ The condition is progressive in many cases, however, and when symptoms become intractable, patients are often confronted with the dilemma of living with persistent pain and functional impairment or electing one

Abbreviations used in this paper: $\mathrm{AP}=$ anteroposterior; $\mathrm{MR}=$ magnetic resonance; NSAID = nonsteroidal antiinflammatory drug; ZCQ = Zürich Claudication Questionnaire. of several surgical options, including decompressive laminectomy with or without fusion. ${ }^{3}$ Interspinous process decompression is a minimally invasive surgical procedure that offers an intermediate step in the continuum of care for these patients. With this procedure, a spacer is inserted between contiguous spinous processes and placed posterior to the neural elements, which has the advantage of preserving native anatomical structures and minimizing the risk of neural injury. Consequently, undergoing this procedure does not eliminate the possibility for more invasive surgery at a later time if severe symptoms persist.

\section{The X STOP Device}

Mechanisms of Action. The X STOP (St. Francis Medical Technologies, Inc.), a commercially available interspinous process decompression device, has been shown to be safe and effective for the treatment of patients with moderately impaired physical function whose symptoms are exacerbated in extension and relieved in flexion. ${ }^{15}$ The $\mathrm{X}$ STOP reduces extension at the symptomatic level(s), preventing narrowing of the spinal canal while preserving natural, unrestricted motion in flexion, lateral bending, and axial rotation. ${ }^{8-10}$ Although neuroimaging studies such as MR imaging are required to confirm the diagnosis of lumbar spinal stenosis, the degree of canal or foraminal narrowing is only weakly correlated with the severity of presenting symptoms. . $^{4-12,13}$ Consequently, selection of patients for interspinous process decompression with the X STOP device necessitates careful assessment of physical symptoms and walking distances to establish a primary diagnosis of lumbar spinal stenosis. 
Indications. The X STOP system is indicated for treatment of patients 50 years of age or older who are suffering from neurogenic intermittent claudication secondary to a confirmed diagnosis of lumbar spinal stenosis. Confirmation is based on x-ray film, MR imaging, and/or computed tomography evidence of thickened ligamentum flavum, narrowed lateral recess, and/or central canal narrowing. The X STOP is indicated for patients with moderately impaired physical function who experience relief in flexion from their symptoms of leg/buttock/groin pain, with or without back pain, and who have undergone a regimen of at least 6 months of nonsurgical treatment. The X STOP may be implanted at one or two lumbar levels in patients in whom surgical treatment is indicated at no more than two levels.

Contraindications. The following conditions are contraindications for this procedure: 1) an allergy to titanium or titanium alloy; 2) spinal anatomy or disease that would prevent implantation of the device or cause it to be unstable in situ (this includes significant instability of the lumbar spine [for example, isthmic spondylolisthesis greater than Grade 1.0]; an ankylosed segment at the affected level(s); acute fracture of the spinous process or pars interarticularis; and significant scoliosis [Cobb angle $\geq 25^{\circ}$ ]); 3 ) cauda equina syndrome, defined as neural compression causing neurogenic bowel or bladder dysfunction; 4) diagnosis of severe osteoporosis, defined as bone mineral density (based on dual-energy x-ray absorptiometry scan or some comparable study) in the spine or hip that is more than 2.5 standard deviations below the mean of adult normal individuals in the presence of one or more fragility fractures; and 5) active systemic infection or infection localized to the site of implantation.

\section{Illustrative Cases}

Six illustrative cases are described that encompass the types of patients who are eligible for interspinous process decompression treatment with the X STOP system under the approved indications for use. All patients described were enrolled in a pivotal randomized controlled trial of the safety and effectiveness of the X STOP device in the treatment of neurogenic intermittent claudication associated with lumbar spinal stenosis. ${ }^{15}$ Clinically significant improvements over 24 months of follow-up care were defined a priori as specific degrees of quantitative change in the three domains of the ZCQ. ${ }^{11,14,15}$

\section{Case 1: One-Level Spinal Stenosis}

History. This 61-year-old woman presented with lowback pain and concomitant radiating pain to both buttocks and legs that had continued for 7 months, with increasing severity of claudication symptoms. She was unable to walk or stand, and she could not sit without pain (she was wheelchair bound). Her pain was unresponsive to conservative treatment, including four epidural steroid injections, Celebrex, and Tylenol with codeine, which were administered in the course of 6 months.

Diagnosis. Neurogenic intermittent claudication and spinal stenosis with facet joint hypertrophy and a synovial facet joint cyst at L3-4 (Fig. 1A and B) was diagnosed.

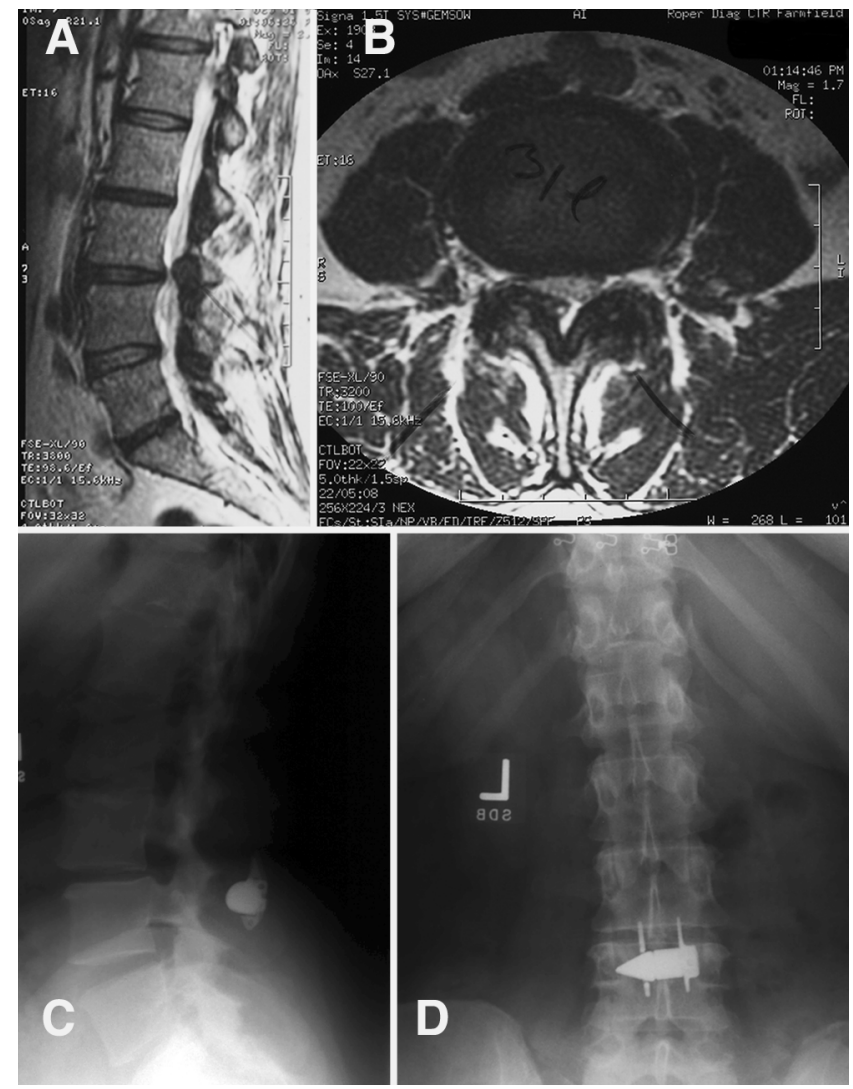

FIG. 1. Case 1. Preoperative sagittal (A) and axial (B) MR images obtained in a patient with one-level (L3-4) lumbar spinal stenosis. Note the focal narrowing at the L3-4 level in the sagittal view, and the thickened ligamentum flavum and narrow lateral recesses in the axial view. Postoperative lateral (C) and AP (D) $\mathrm{x}$ ray films of the patient after treatment with a one-level X STOP device at the L3-4 level.

Treatment. One-level X STOP implantation at L3-4 (12 $\mathrm{mm})$ was performed, and there were no complications (Fig. 1C and D). The total operating time was 90 minutes, intraoperative blood loss was $50 \mathrm{ml}$, and the patient was discharged 24 hours postoperatively.

Outcome. Presenting symptoms were relieved immediately after surgery, with the patient demonstrating sustained, clinically significant improvement in symptom severity and degree of functional impairment through 24 months of follow up. Treatment success was confirmed based on ZCQ scores. The patient's gait is normal and her ambulation is pain free. Radiographic assessments performed at 6,12 , and 24 months postoperatively revealed no change in the X STOP positioning, no spondylolisthesis, and no changes to the spinous process, laminae, or facets.

\section{Case 2: Two-Level Spinal Stenosis}

History. This 50-year-old man presented with low-back pain featuring some radiating pain to both legs, which had been occurring sporadically for 5 to 10 years. His pain was partially responsive to a 6-week course of physical therapy, followed by resumption of symptoms. Four epidural injections of steroids were required over a 2-year period to manage symptoms adequately. 


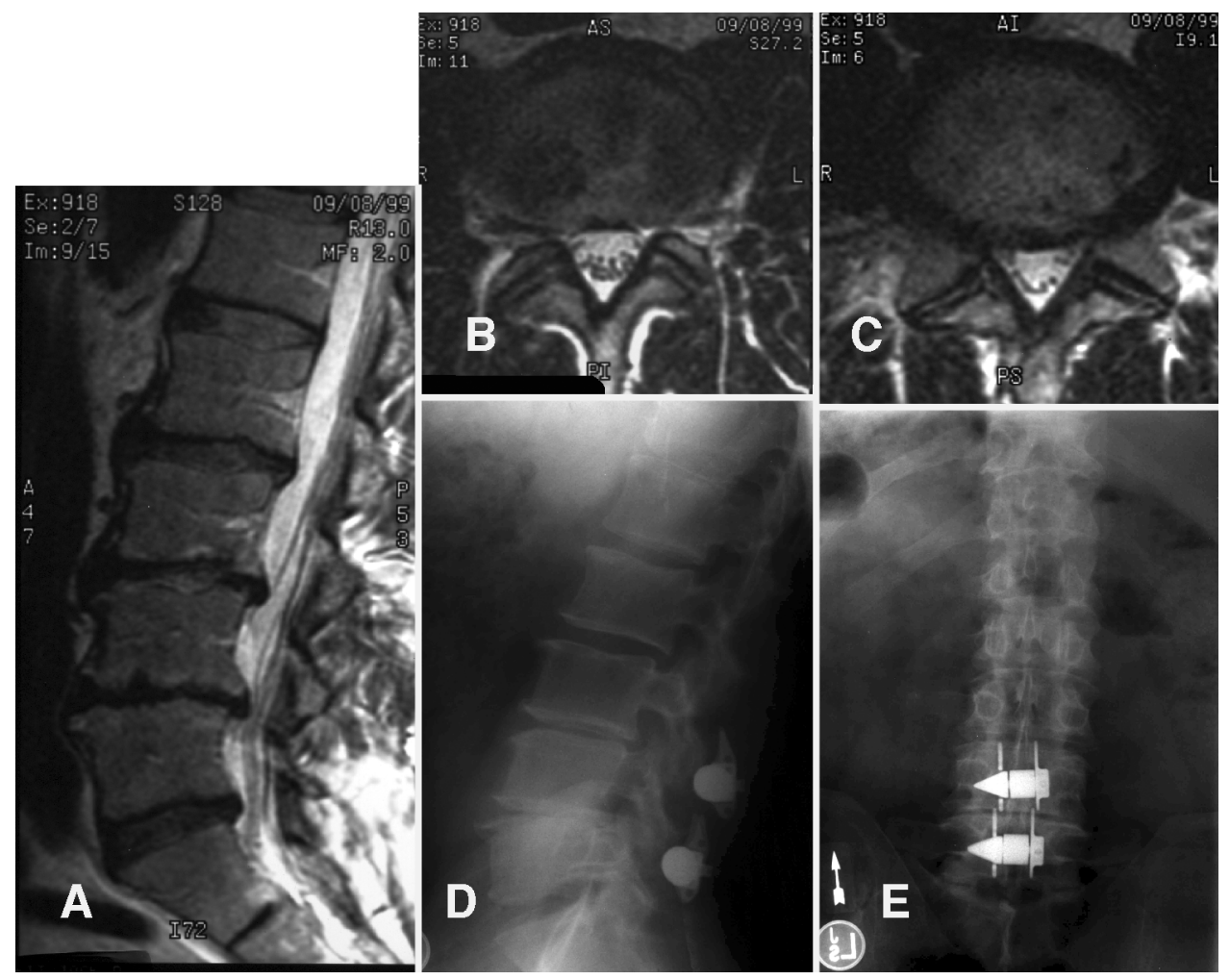

FIG. 2. Case 2. Preoperative sagittal (A) and axial L3-4 (B) and L4-5 (C) MR images obtained in a patient with twolevel (L3-4 and L4-5) lumbar spinal stenosis. Note the focal narrowing at the L3-4 and L4-5 levels in the sagittal view, and the narrow lateral recesses in the axial views. Postoperative lateral (D) and AP (E) X-ray films of the patient after treatment with two-level X STOPs at the L3-4 and L4-5 levels.

Diagnosis. Degenerative disc disease with disc space narrowing and lateral recess stenosis at L3-4 and L4-5 (Fig. 2A-C) was diagnosed.

Treatment. Two-level X STOP implantation at L3-4 (12 $\mathrm{mm})$ and L4-5 (12 $\mathrm{mm})$ was performed after induction of conscious sedation, and no complications occurred (Fig. $2 \mathrm{D}$ and $\mathrm{E}$ ). The total operating time was 95 minutes, intraoperative blood loss was $50 \mathrm{ml}$, and the patient was discharged 24 hours postoperatively.

Outcome. Presenting symptoms were relieved at 6 weeks posttreatment, with the patient demonstrating sustained, clinically significant improvement in symptom severity and degree of functional impairment through 24 months of follow up. Treatment success was confirmed based on ZCQ scores. The patient showed progressive improvement at each evaluation and has resumed previous activities such as golfing. He reported satisfaction with the degree of improvement and would repeat the procedure. Radiographic assessments performed at 6, 12, and 24 months postoperatively revealed no change in the X STOP positioning, no spondylolisthesis, and no changes to the spinous process, laminae, or facets.

\section{Case 3: Mild Spinal Stenosis on Neuroimaging}

History. This 73-year-old man presented with left leg pain that had been occurring sporadically for more than 1 year. His pain was unresponsive to conservative treatment, including NSAIDs and physical therapy.
Diagnosis. A Grade I spondylolisthesis at the L4/5 juncture, degenerative facet disease at L4-5 and L5-S1, moderate spinal stenosis at L3-4, mild stenosis at L4-5 with multilevel degenerative disc disease, and mild right scoliosis (Fig. 3A-C) were diagnosed.

Treatment. Two-level X STOP implantation at L3-4 (12 $\mathrm{mm})$ and L4-5 (10 $\mathrm{mm})$ was performed after induction of local anesthesia, and there were no complications (Fig. 3D and $\mathrm{E})$. The total operating time was 45 minutes, intraoperative blood loss was $10 \mathrm{ml}$, and the patient was discharged 24 hours postoperatively.

Outcome. Presenting symptoms were relieved at 6 weeks posttreatment, with the patient demonstrating sustained, clinically significant improvement in symptom severity and degree of functional impairment through 24 months of follow up. Treatment success was confirmed based on the ZCQ score. The patient reported no residual back or leg pain, numbness, or tingling in the lower extremities. Radiographic assessments performed at 6,12 , and 24 months postoperatively revealed no change in the $\mathrm{X}$ STOP positioning, and no changes to the spinous process, laminae, or facets.

\section{Case 4: Severe Spinal Stenosis on Neuroimaging}

History. This 73-year-old woman presented with intermittent left buttock pain with concomitant radiating left leg pain that had lasted for 6 months and was becoming increasingly more frequent and severe. Her pain was unre- 


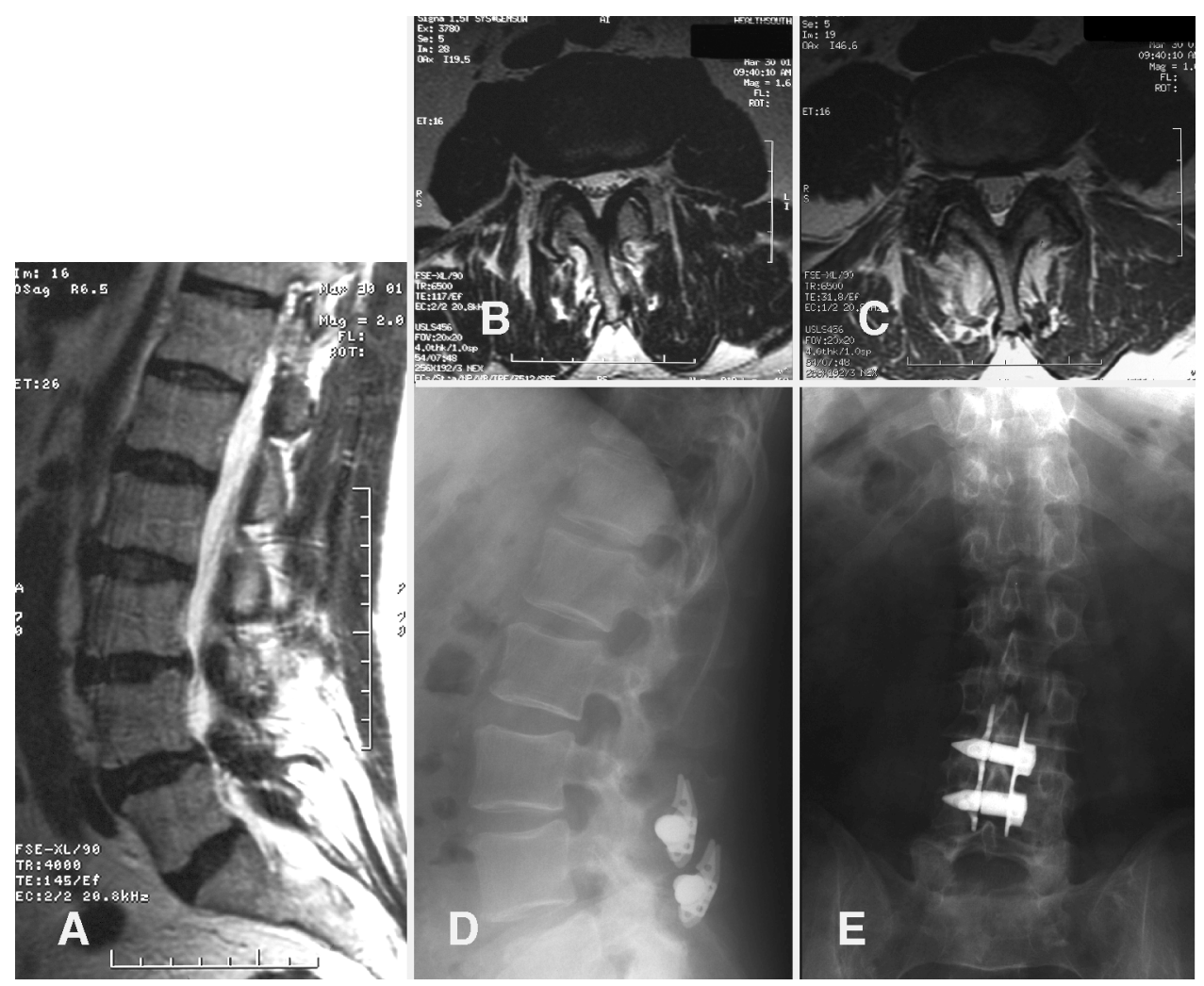

FIG. 3. Case 3. Preoperative sagittal (A) and axial L3-4 (B) and L4-5 (C) MR images obtained in a patient with twolevel (L3-4 and L4-5) lumbar spinal stenosis. Note the relatively mild neuroimaging-confirmed central stenosis at the L3-4 and L4-5 levels. Postoperative lateral (D) and AP (E) X-ray films of the patient after treatment with two-level X STOPs at the L3-4 and L4-5 levels.

sponsive to conservative treatment with pain medications, including daily Ultram, Celebrex, and Tylenol as well as three epidural steroid injections, physical therapy, and NSAIDs, which had yielded minimal relief.

Diagnosis. Severe L4-5 spinal stenosis secondary to Grade I spondylolisthesis associated with a mild disc bulge was diagnosed based on MR imaging findings. Additionally, less severe L3-4 spinal stenosis secondary to mild anterior spondylolisthesis, and L2-3 canal narrowing (Fig. 4A-C) were found.

Treatment. Two-level X STOP implantation at L4-5 (12 $\mathrm{mm})$ and L3-4 (12 $\mathrm{mm})$ was performed after induction of local anesthesia, and no complications occurred (Fig. 4D and $\mathrm{E}$ ). The total operating time was 45 minutes, intraoperative blood loss was $10 \mathrm{ml}$, and the patient was discharged less than 24 hours postoperatively.

Outcome. Presenting symptoms were relieved at 6 weeks posttreatment, with the patient demonstrating sustained, clinically significant improvement in symptom severity and degree of functional impairment through 24 months of follow up. Treatment success was confirmed based on ZCQ scores. The patient's leg and buttock pain was resolved and her quality of life improved. Radiographic assessments performed at 6, 12, and 24 months postoperatively revealed no change in the X STOP positioning, and no changes to the spinous process, laminae, or facets.

\section{Case 5: Spondylolisthesis With Spinal Stenosis}

History. This 82-year-old woman presented with bilateral lower-extremity pain that had been increasing in severity over a 5-month period. She was unable to walk more than two blocks without pain, but experienced pain relief when sitting. Her pain was unresponsive to conservative treatment, including epidural steroid injections and NSAIDs.

Diagnosis. Moderate spinal stenosis at L3-4, moderate to severe stenosis at L4-5, and mild spondylolisthesis at the L4/5 juncture with associated degenerative disc disease (Fig. 5A) were diagnosed.

Treatment. Two-level X STOP implantation was performed at L3-4 (10 mm) and L4-5 (10 mm) (Fig. 5B). The total operating time was 75 minutes, intraoperative blood loss was $10 \mathrm{ml}$, and the patient was discharged 24 hours postoperatively.

Outcome. Presenting symptoms were relieved at 6 weeks posttreatment, with the patient demonstrating sustained, clinically significant improvement in symptom severity and degree of functional impairment through 24 months of follow up. Treatment success was confirmed based on ZCQ scores. The patient reported no pain, no difficulty with walking, standing, or sitting, and a very good to excellent quality of life. There was lingering mild discomfort with lifting maneuvers. Radiographic assess- 


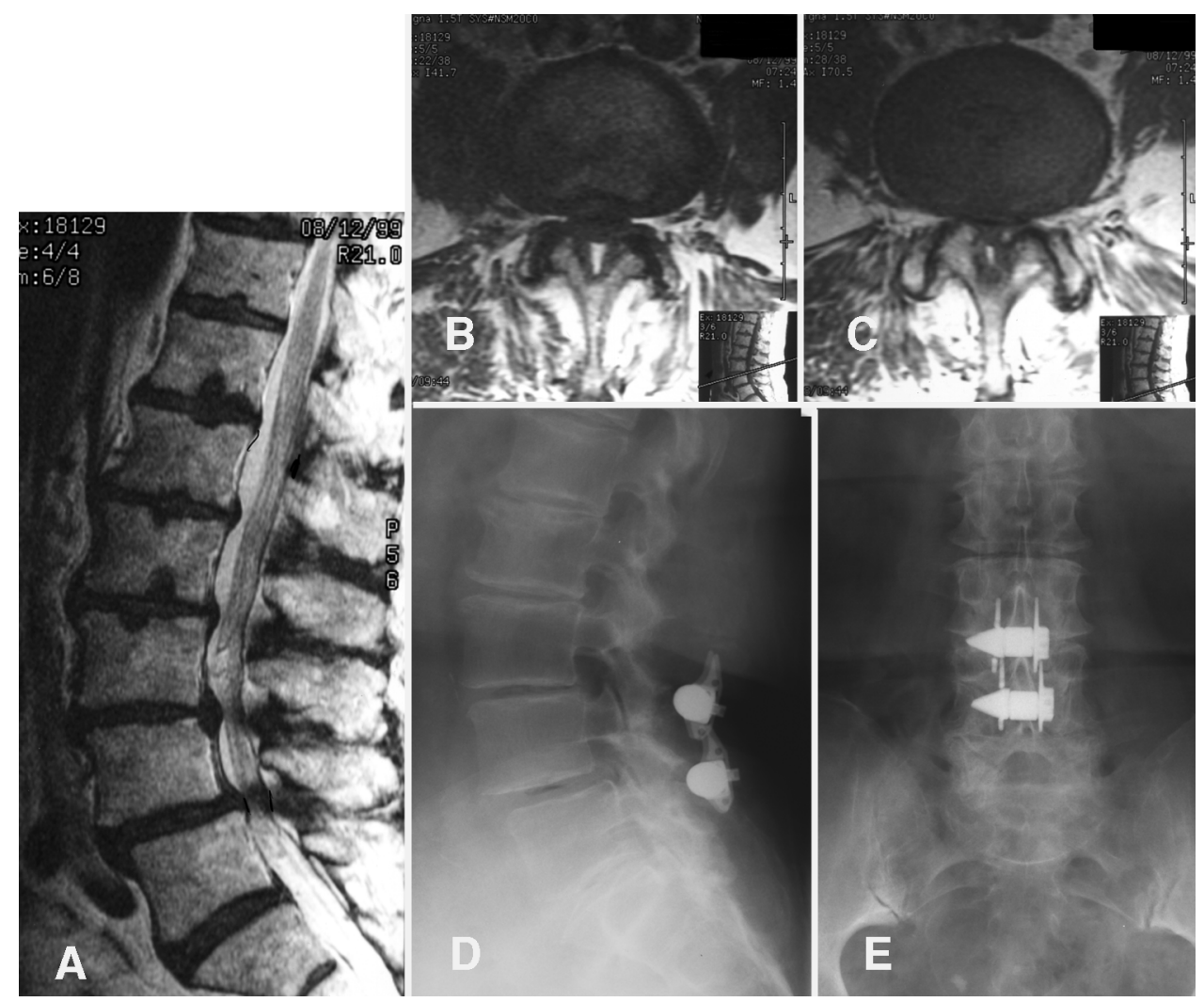

FIG. 4. Case 4. Preoperative sagittal (A) and axial L3-4 (B) and L4-5 (C) MR images obtained in a patient with twolevel, L3-4 and L4-5 lumbar spinal stenosis. Note the relatively severe neuroimaging-confirmed central stenosis at the L4-5 levels and the thickened ligament flavum at L3-4 and L4-5. Postoperative lateral (D) and AP (E) X-ray films of the patient after treatment with two-level X STOPs at the L3-4 and L4-5 levels.

ments performed at 6,12 , and 24 months postoperatively revealed no change in the X STOP positioning, and no changes to the spinous process, laminae, or facets.

\section{Case 6: Scoliosis With Spinal Stenosis}

History. This 70-year-old man presented with frequently severe leg pain that had lasted for 6 months, causing interference in his activities of daily living and his work life. His pain was unresponsive to conservative treatment, including pain medications (Vicodin), NSAIDs, and two epidural steroid injections.

Diagnosis. Mild scoliosis $\left(20^{\circ}\right)$ and spondylolisthesis with spinal stenosis at L4-5 (Fig. 6) were diagnosed.

Treatment. One-level X STOP implantation at L4-5 (6 $\mathrm{mm}$ ) was performed after induction of local anesthesia, and no complications occurred (Fig. 6). The total operating time was 44 minutes, intraoperative blood loss was 60 $\mathrm{ml}$, and the patient was discharged less than 24 hours postoperatively.

Outcome. Relative to pretreatment findings, the patient demonstrated sustained, clinically significant improvement in symptom severity and degree of functional impairment through 24 months of follow up. Treatment success was confirmed based on ZCQ scores. The patient reported no residual leg pain and was able perform activities of daily living and work without difficulty. Radiographic assessments performed at 6,12, and 24 months postoperatively showed no changes in the X STOP position, no metallosis, and no changes to the spinous process, laminae, or facets. At 24 months the follow-up x-ray films indicated 5\% spondylolisthesis at the treated level, suggesting little or no change from preoperative status.

\section{Discussion}

Interspinous process decompression with the X STOP device offers an intermediate step in the continuum of care for patients with neurogenic intermittent claudication associated with lumbar spinal stenosis. Afflicted patients whose pain is unresponsive to at least 6 months of nonsurgical conservative management are unlikely to show clinically significant improvement with continuation of this form of care. In a randomized controlled trial, $\mathrm{Zu}$ cherman et al. ${ }^{15}$ demonstrated that only approximately $5 \%$ of participants assigned to continuation of conservative treatment met the a priori-defined treatment success criteria for the study after 24 months of follow up, with approximately $28 \%$ requiring decompressive laminectomy due to unresolved symptoms. This contrasted sharply with the findings in patients treated with the X STOP device, approximately $48 \%$ of whom satisfied the three ZCQ success criteria, with $7 \%$ requiring laminectomy. ${ }^{15}$ To put these success rates in perspective, Tuli et al. ${ }^{14}$ reported that $47 \%$ of patients who had undergone laminectomy in a multicenter study met the same ZCQ success criteria. 

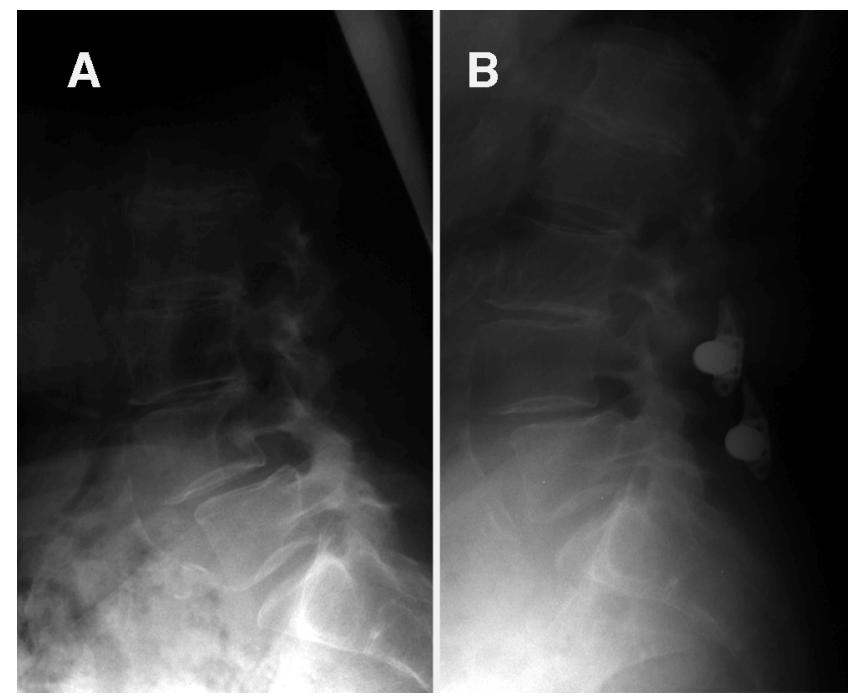

FIG. 4. Case 5. Preoperative (A) and postoperative (B) lateral $\mathrm{x}^{-}$ ray films obtained in a patient with Grade I spondylolisthesis at the L4-5 level. The X STOP devices were implanted at the L3-4 and L4-5 levels (note the stable L4-5 level following treatment).

The case material provided in this report illustrates the type and range of patients most likely to demonstrate clinically meaningful improvements after treatment with the $\mathrm{X}$ STOP device. With careful patient selection for this procedure, it is highly likely that progression to more invasive surgical interventions, such as decompressive laminectomy, will be avoided or delayed substantially. Cases 1 and 2 represent typical patient types with stenotic symptoms at one and two levels, respectively. It can be expected that clinical improvements achieved with the $\mathrm{X}$ STOP system are durable. Kondrashov et al. ${ }^{7}$ demonstrated that the success rate among patients treated with $\mathrm{X}$ STOP devices at one and two levels was $78 \%$ at a mean of 4.2 years postoperatively, which is consistent with the intermediate-term ( 1 and 2 years) results of Zucherman et al. ${ }^{15,16}$

Treatment with the X STOP device provides effective amelioration of stenotic symptoms regardless of the degree of central canal stenosis. Cases 3 and 4 provide contrasting illustrations of patients with mild and severe neuroimaging-determined stenosis, respectively. Appropriate distraction and decompression was achieved with the $\mathrm{X}$ STOP system, and both patients realized clinically significant improvements through 24 months of follow up. Similarly, Herno and colleagues ${ }^{4,5}$ identified only weak correlations between the degree of decompression achieved with laminectomy and the amount of resolution of neurogenic symptoms.

The X STOP device is appropriate for use in patients who have moderately severe neurogenic symptoms associated with mild lumbar degenerative spondylolisthesis (Grade I), as illustrated by Case 5. Anderson et al. ${ }^{1}$ demonstrated overall clinical success rates of approximately $63 \%$ in patients with mild spondylolisthesis who were treated with the X STOP device and $13 \%$ in those who received nonsurgical conservative care.

Case 6 illustrates that patients with mild scoliosis $(<$
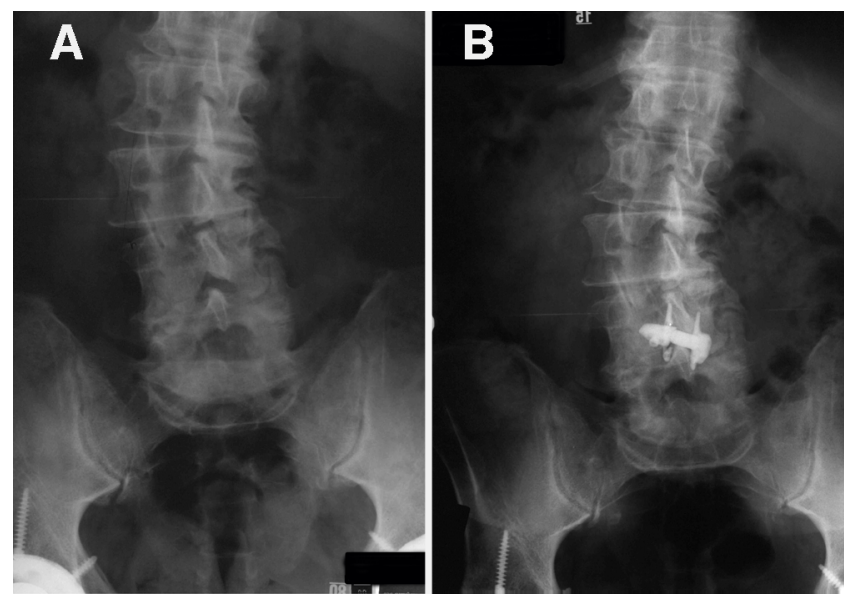

FIG. 5. Case 6. Preoperative (A) and postoperative (B) AP x-ray films obtained in a patient with degenerative lumbar scoliosis and neurogenic intermittent claudication secondary to lumbar spinal stenosis. Note the stable curve after treatment.

$25^{\circ}$ ) and appropriate clinical symptoms also may benefit from interspinous process decompression with the $\mathrm{X}$ STOP device. This patient showed excellent symptomatic improvement in neurogenic signs and physical functioning through 24 months of follow up.

Carefully selected patients with established neurogenic intermittent claudication whose pain is unresponsive to conservative care and in whom confirmatory imaging evidence of lumbar spinal stenosis is found realize discernible clinical benefits from interspinous process decompression with the X STOP device. These patients have moderate functional impairment and are unlikely to recover spontaneously with further nonsurgical care. Many would probably be candidates for more invasive spinal surgery to alleviate their symptoms. The X STOP device offers a minimally invasive intermediate step in the continuum of care for these patients that yields long-term, durable clinical improvement.

\section{References}

1. Anderson PA, Tribus CB, Kitchel SH: Treatment of neurogenic claudication by interspinous decompression: application of the $\mathrm{X}$ STOP device in patients with lumbar degenerative spondylolisthesis. J Neurosurg Spine 4:463-471, 2006

2. Arbit E, Pannullo S: Lumbar stenosis: a clinical review. Clin Orthop Relat Res 384:137-143, 2001

3. Fritz JM, Delitto A, Welch WC, Erhard RE: Lumbar spinal stenosis: a review of current concepts in evaluation, management, and outcome measurements. Arch Phys Med Rehabil 79:700-708, 1998

4. Herno A, Airaksinen O, Saari T: Computed tomography after laminectomy for lumbar spinal stenosis. Patients' pain patterns, walking capacity, and subjective disability had no correlation with computed tomography findings. Spine 19:1975-1978, 1994

5. Herno A, Saari T, Suomalainen O, Airaksinen O: The degree of decompressive relief and its relation to clinical outcome in patients undergoing surgery for lumbar spinal stenosis. Spine 24:1010-1014, 1999

6. Jalovaara $\mathrm{P}$, Lahde $\mathrm{S}$, Iikko E, Niinimaki T, Puranen J, Lindholm RV: The significance of residual stenosis after decompression for lumbar spinal stenosis. Ann Chir Gynaecol 78:304-308, 1989 


\section{Interspinous process decompression with the X STOP device}

7. Kondrashov DG, Hannibal M, Hsu KY, Zucherman JF: Interspinous process decompression with the X-STOP device for lumbar spinal stenosis: a 4-year follow-up study. J Spinal Disord Tech 19:323-327, 2006

8. Richards JC, Majumdar S, Lindsey DP, Beaupre GS, Yerby SA: The treatment mechanism of an interspinous process implant for lumbar neurogenic intermittent claudication. Spine 30: 744-749, 2005

9. Siddiqui M, Karadimas E, Nicol M, Smith FW, Wardlaw D: Effects of X-STOP device on sagittal lumbar spine kinematics in spinal stenosis. J Spinal Disord Tech 19:328-333, 2006

10. Siddiqui M, Nicol M, Karadimas E, Smith F, Wardlaw D: The positional magnetic resonance imaging changes in the lumbar spine following insertion of a novel interspinous process distraction device. Spine 30:2677-2682, 2005

11. Stucki G, Daltroy L, Liang MH, Lipson SJ, Fossel AH, Katz $\mathrm{JN}$ : Measurement properties of a self-administered outcome measure in lumbar spinal stenosis. Spine 21:796-803, 1996

12. Szpalski M, Gunzburg R: Lumbar spinal stenosis in the elderly: an overview. Eur Spine J 12 (2 Suppl): S170-s175, 2003

13. Thomas NW, Rea GL, Pikul BK, Mervis LJ, Irsik R, McGregor JM: Quantitative outcome and radiographic comparisons between laminectomy and laminotomy in the treatment of acquired lumbar stenosis. Neurosurgery 41:567-575, 1997
14. Tuli SK, Yerby SA, Katz JN: Methodological approaches to developing criteria for improvement in lumbar spinal stenosis surgery. Spine 31:1276-1280, 2006

15. Zucherman JF, Hsu KY, Hartjen CA, Mehalic TF, Implicito DA, Martin MJ, et al: A multicenter, prospective, randomized trial evaluating the X STOP interspinous process decompression system for the treatment of neurogenic intermittent claudication: two-year follow-up results. Spine 30:1351-1358, 2005

16. Zucherman JF, Hsu KY, Hartjen CA, Mehalic TF, Implicito DA, Martin MJ, et al: A prospective randomized multi-center study for the treatment of lumbar spinal stenosis with the $\mathrm{X}$ STOP interspinous implant: 1-year results. Eur Spine J 13: 22-31, 2004

Manuscript received November 1, 2006.

Accepted in final form November 20, 2006.

This study was supported by St. Francis Medical Technologies, Inc, in Alameda, California.

Address reprint requests to: Carl Lauryssen, M.D., Tower Orthopedics and Neurosurgical Spine, 8670 Wilshire Boulevard, Suite 202, Beverly Hills, California 90211. email: DrCl@ Olympiamc.com. 